\title{
High-performance midinfrared quantum cascade lasers
}

\author{
Federico Capasso, FELLOW SPIE \\ Harvard University \\ School of Engineering and Applied Sciences \\ Cambridge, Massachusetts 02138
}

\begin{abstract}
The design and operating principles of quantum cascade lasers (QCLs) are reviewed along with recent developments in high-power cw and broadband devices. Cw power levels of several watts at room temperature have been achieved at $4.6-\mu \mathrm{m}$ wavelength; broadband single-mode tuning $\left(\approx 400 \mathrm{~cm}^{-1}\right)$ has been achieved using an external-cavity QCL with a grating as a tuning element. An alternative approach, consisting of a monolithically integrated array of single-mode QCLs individually currentdriven by a microcontroller, has led to broadband single-mode tuning over a range of $200 \mathrm{~cm}^{-1}$ without requiring the use of moving parts. This spectrometer on a chip holds promise for high-brightness compact tracegas sensors capable of operating in harsh environments. (c) 2010 Society of Photo-Optical Instrumentation Engineers. [DOI: 10.1117/1.3505844]
\end{abstract}

Subject terms: quantum cascade lasers; laser arrays; high power; broadband; single mode.

Paper 100467SSR received Jun. 6, 2010; revised manuscript received Aug. 12, 2010; accepted for publication Aug. 18, 2010; published online Nov. 22, 2010.

\section{Introduction}

Laser diodes emitting at wavelengths ranging from the near infrared to the visible are the workhorses of widespread technologies such as optical communications, optical recording (CD players, etc.), supermarket scanners, laser printers, fax machines, and laser pointers. The operating principle of these lasers is fundamentally simple: electrons and holes are electrically injected into an active region made of semiconductor materials, where they recombine, giving off laser photons of wavelength close to the bandgap of the active region. A corollary of this is that if one wishes to build diode lasers emitting at very different wavelengths, one needs to choose materials with widely different band gaps and therefore very different electronic and optical properties-for example, quaternary alloys of indium, gallium, phosphorus, and arsenic for lasers emitting at telecom wavelengths around 1.3 and $1.5 \mu \mathrm{m}$, and alloys of gallium, indium, aluminum, and nitrogen for blueemitting lasers. In spite of its simplicity and generality, the diode laser principle has proven hard to extend to the midinfrared while maintaining the same level of performance of its shorter-wavelength counterparts. The reliance on the bandgap for light emission turns into a severely limiting factor at midinfrared wavelengths, particularly across a large portion $(3.5$ to $20 \mu \mathrm{m})$ of the so-called molecular fingerprint region, where most molecules absorb light. The reason is that as the bandgap shrinks in a semiconductor laser, its operation becomes much more critical with respect to the maximum operating temperature, temperature stabilization required to avoid thermal runaway effects, and thermal recycling. To these problems one should add that, as the band gap shrinks, chemical bonds become weaker; this increased material softness facilitates the introduction of defects during growth and device fabrication, making diode lasers less reliable and reducing device yields.

For example, semiconductor laser diodes made of lead salts ${ }^{1,2}$ and emitting in the mid-IR, which have been used for

0091-3286/2010/\$25.00 @ 2010 SPIE many years in tunable laser spectroscopy, suffer from all of these limits. Lead-salt lasers have limited power (at most a few milliwatts of peak and continuous-wave power), have a small continuous single-mode tuning range, and have yet to operate at room temperature. They also suffer from spectral degradation and reliability problems associated with thermal cycling.

The QCL avoids the operating principle of conventional semiconductor lasers by relying on a radically different process for light emission, which is independent of the band gap. Instead of using opposite-charge carriers in semiconductors (electrons and holes) at the bottom of their respective conduction and valence bands, which recombine to produce light of frequency $v \approx E_{g} / h$, where $E_{g}$ is the energy band gap and $h$ is Planck's constant, QCLs use only one type of charge carriers (electrons), which undergo quantum jumps between energy levels $E_{n}$ and $E_{n-1}$ to create a laser photon of frequency $\left(E_{n}-E_{n-1}\right) / h$. These energy levels do not exist naturally in the constituent materials of the active region, but are artificially created by structuring the active region into quantum wells of nanometric thickness. The motion of electrons perpendicular to the layer interfaces is quantized and characterized by energy levels whose difference is determined by the thickness of the wells and by the height of the energy barriers separating them. The implication of this new approach, based on decoupling light emission from the band gap by utilizing instead optical transitions between quantized electronic states, are many and far reaching, amounting to a laser with entirely different operating characteristics from laser diodes and far superior performance and functionality.

This review will be limited to mid-IR QCLs. While there has been substantial progress in far-IR (also known as terahertz) QCLs, their use in spectroscopy and chemical sensing has been as yet limited, unlike their counterparts in the mid-IR. The main reason is their very limited tuning range, in addition to the fact that they have to be cryogenically cooled. There indeed appear to be fundamental limits associated with the physics of transport in QCLs that will prevent 
room-temperature operation in the far IR, although Peltiercooled devices might be possible.

The first QCL was invented and demonstrated ${ }^{3}$ at Bell Labs in 1994 by Faist, Capasso, Sivco, Sirtori, Hutchinson, and Cho. The first prototypes operated only in pulsed mode at a maximum temperature of $90 \mathrm{~K}$. Advancements in band engineering and waveguide designs led within a few years to many important achievements: pulsed room-temperature operation, continuous wave (cw) and single-mode operation, extension of the operating wavelength to values as short as $3.5 \mu \mathrm{m}$ and as long as $19 \mu \mathrm{m}$, and the first applications to spectroscopy and chemical sensing. For reviews of these and other developments see Refs. 4 and 5.

\section{Quantum Cascade Laser Design}

In QCLs, unlike in a laser diode, an electron remains in the conduction band after emitting a laser photon. The electron can therefore easily be recycled by being injected into an adjacent identical active region, where it emits another photon, and so forth. To achieve this cascading emission of photons, active regions are alternated with doped electron injectors and an appropriate bias voltage is applied. The active-regioninjector stages of the QC laser give rise to an energy staircase in which photons are emitted at each of the steps. The number of stages typically ranges from 20 to 35 for lasers designed to emit in the 4 - to $8-\mu \mathrm{m}$ range, but working lasers can have as few as one or as many as 100 stages. This cascade effect is responsible for the very high power that QCLs can attain.

Figure 1 shows a typical energy diagram of two stages of a QCL designed to operate at wavelength $\lambda=7.5 \mu \mathrm{m}$, which serves to illustrates the key operating principle. Note that each stage comprises an electron injector and an active region. The latter contains three quantized states; the laser transition is defined by the energy difference between states 3 and 2, which is determined primarily by the chosen thickness of the two wider wells. A population inversion between levels 3 and 2 is required for laser action. This translates to a requirement that the electron relaxation time between levels 3 and 2 should be substantially longer than the lifetime of state 2. To achieve that, the lowest level 1 is positioned about an optical phonon energy $(\approx 34 \mathrm{meV})$ below level 2 ; this ensures that electrons in the latter state rapidly scatter by

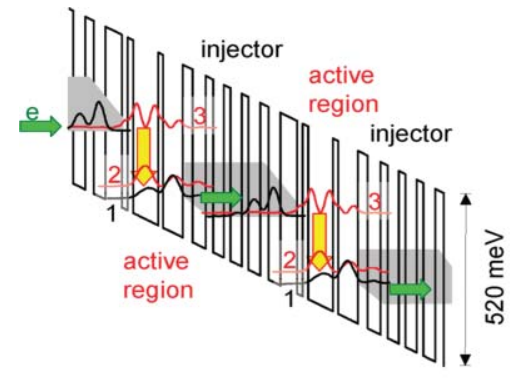

(a)

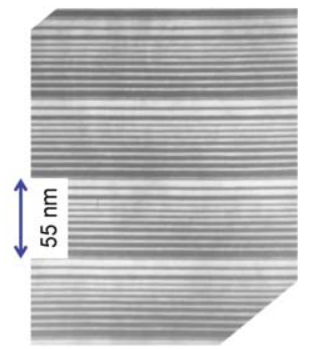

(b)
Fig. 1 (a) Energy diagram of a quantum cascade laser emitting at $\lambda=7.5 \mu \mathrm{m}$. Each stage (injector plus active region) is $55 \mathrm{~nm}$ thick. The energy levels and the corresponding probability distributions obtained from solving Schrödinger's equation are shown. The energy well and barriers are made of AllnAs and GalnAs alloys latticematched to InP, respectively. (b) Transmission electron microscope picture of a portion of the structure. The white and black contrast regions represent the well and barriers, respectively. (Color online only.) emission of an optical phonon to energy level 1. Because of its resonant nature, this process is very fast, characterized by a relaxation time of the order of 0.1 to $0.2 \mathrm{ps}$. Electrons in level 3 have instead a substantially longer lifetime because of the much larger energy difference between states 3 and 2, so that the electron-phonon scattering process between the latter states is nonresonant. To achieve lasing, however, one must also suppress the unwanted escape route by tunneling from state 3 to states on the right-hand side, which form a broad quasicontinuum. Such escape would reduce the level 3 population. To prevent this occurrence, one designs the injector of the next stage as a superlattice with an electronic density of states such that at the energy corresponding to $E_{3}$ there is no resonant electronic state, but rather a region of low density of states known as a minigap. Notice in contrast the dense manifold of states (a miniband) facing levels 2 and 1, which favors efficient electron extraction from the active region. Finally, note that electrons are injected into the upper laser level by a process known as resonant tunneling, which ensures highly selective injection when the applied voltage is increased above a certain value. Figure 1 also shows a transmission electron micrograph of the layer structure of an exemplary QCL. The dark layers correspond to the AlInAs barrier layers, and the light gray layers to the GaInAs quantum wells.

For wavelengths greater than $\approx 5 \mu \mathrm{m}$, the alloy compositions for the wells and barriers are chosen to be latticematched to the InP substrate, i.e., with the same lattice constant $\left(\mathrm{Al}_{0.48} \mathrm{In}_{0.52} \mathrm{As}\right.$ and $\left.\mathrm{In}_{0.47} \mathrm{Ga}_{0.53} \mathrm{As}\right)$. The quantum-well barrier height for these compositions is the conduction-band discontinuity between the two alloys, i.e., $\Delta E_{c}=0.52 \mathrm{eV}$. For operating wavelengths several tenths of a micron shorter than $5 \mu \mathrm{m}$, the upper laser state moves up in energy, so that the barrier for thermal activation of electrons is reduced. In $\mathrm{cw}$ operation at room temperature, this process, together with a similar one for electrons in the injector regions, becomes a limiting factor for the temperature performance (maximum operating temperature and maximum optical power at room temperature or above), because the active region can reach temperatures substantially higher than the laser mount by tens of kelvins. This problem is greatly reduced by introducing strained AlInAs/GaInAs heterostructures, i.e., higher $\mathrm{Al}$ composition for the barrier and lower In content for the quantum wells. In this way, barrier heights in the $0.7-$ to $0.80-\mathrm{eV}$ range can be achieved, which suppresses electron leakage over the barriers. All reported ${ }^{6-9}$ high-performance $\mathrm{cw}$ roomtemperature QCLs operating at $\lambda \leq 5.2 \mu \mathrm{m}$ used such strained heterojunctions. Another critical design parameter to improve temperature performance is the energy separation between the lower state of the laser transition (level 2 in Fig. 2) and the injector ground state. It typically should be in excess of $0.1 \mathrm{eV}$ to minimize thermal backfilling of level 2 by electrons in the injector.

In the last few years, major improvement in QCL performance (lower threshold and higher power) was achieved by introducing a so-called double-phonon-resonance design. ${ }^{10}$ The active region of this laser has four quantum wells and three energy levels equally separated by the energy of an optical phonon, instead of the two levels previously discussed. This active-region design results in a larger population inversion, because electrons are more efficiently removed from the lower state of the laser transition and because thermal backfilling of the latter is reduced. 


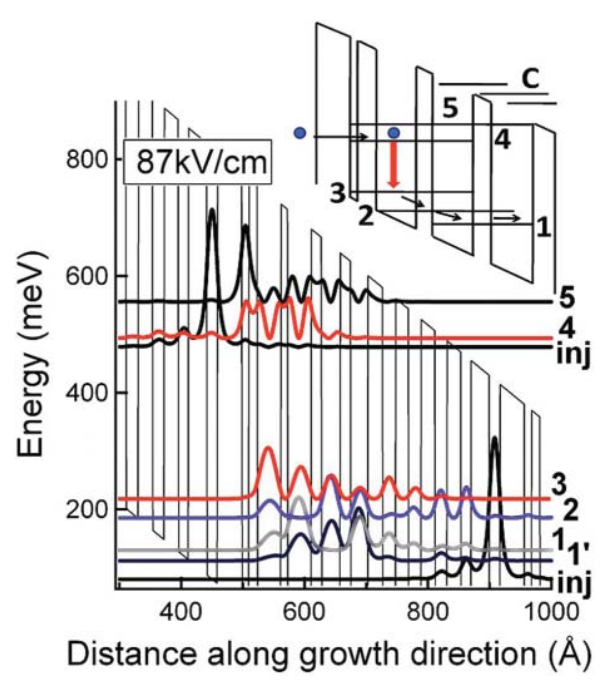

Fig. 2 Band diagram of a QCL structure designed for light emission at $4.6 \mu \mathrm{m}$. Inset shows a schematic of a band diagram corresponding to the two-phonon design approach. From Ref. 8.

\section{High-Power Continuous-Wave Midinfrared QCLs}

QCLs achieve their best performance in the 4.5- to $10-\mu \mathrm{m}$ range. In 2002, cw room-temperature operation was achieved, ${ }^{11}$ and since then continual improvements in the design, material quality, fabrication, and thermal management have led to record optical power of $34 \mathrm{~W}$ pulsed ${ }^{6}$ and $\approx 3.0 \mathrm{~W} \mathrm{cw}^{7,8}$ at room temperature. The corresponding $\mathrm{cw}$ power efficiency for these Fabry-Perot cavity devices was $16.5 \%$ at $4.8 \mu \mathrm{m}$ (Ref. 7 ) and $\approx 13 \%$ at $4.6 \mu \mathrm{m}$ (Ref. 8). The latter was a joint effort between Pranalytica (a company commercializing QCL-based systems) and our group, which has led to products ranging from infrared countermeasure systems (to protect aircraft from shoulder-fired missiles) to beacons and target illuminators (http://pranalytica.com/).

For these devices the active region [consisting of highly strained $(1 \%) \mathrm{In}_{0.67} \mathrm{Ga}_{0.33} \mathrm{As}_{\mathrm{A}} \mathrm{Al}_{0.64} \mathrm{In}_{0.36} \mathrm{As}$ quantum wells and barriers with strain compensation in each stage] was a modification of the standard two-phonon resonance design, called nonresonant extraction, which gives a greater design flexibility. ${ }^{8}$ Figure 2 shows the band diagram of such a structure.

One of the limitations of a two-phonon design's active region is that the fixed energy separation ( 2 optical phonon energies or slightly higher) between levels 3 and 1 (inset of Fig. 2) limits the ability to increase the energy separation between the upper laser state (4) and the parasitic level 5 in short-wavelength lasers designed to operate $\mathrm{cw}$ at room temperature or above. This limited flexibility hampers efforts to optimize high-temperature performance. One way of increasing the structure's design flexibility is to remove the two-phonon resonance condition, i.e., $E_{32}$ and $E_{21}$ both simultaneously equal to $E_{\mathrm{LO}}$, without sacrificing the efficient carrier extraction from the lower laser level.

The nonresonant extraction design circumvents this problem by introducing an additional quantum well in the active region, which creates a state $1^{\prime}$ designed to have a large spatial overlap with state 2 . This allows one to increase the energy separation between the lower laser level 3 and the lowest active region level 1 . The separation $E_{31}$ was increased up to $100 \mathrm{meV}$, further suppressing thermal backfilling of the lower laser level 3. Both levels, 1 and $1^{\prime}$, have a large overlap with level 2. Due to the availability of two parallel transitions for electron relaxation from level 2, the calculated electron lifetime $\tau_{2}$ of state 2 is only $0.34 \mathrm{ps}$, close to typical values for the two-phonon design, despite the fact that $E_{21}=55 \mathrm{meV}$ and $E_{21}=73 \mathrm{meV}$ are significantly above the energy of longitudinal optical phonons. In addition, $\tau_{2}$ was not very sensitive to variation in the active-region layer thicknesses. This allowed us, in addition to increasing $E_{31}$, to also increase $E_{54}$ up to $63 \mathrm{meV}$, while keeping a high matrix element for the laser transition $z_{43}=1.48 \mathrm{~nm}$ and a large upper-laser-level lifetime $\tau_{4}=1.77 \mathrm{ps}$.

The entire structure was grown by molecular beam epitaxy (MBE). The wafer was then processed into a buried heterostructure $(\mathrm{BH})$ geometry. First, a combination of reactive ion etching and wet chemical etching was used to form the ridges, which were then overgrown with semiinsulating iron-doped InP. After conventional substrate thinning, polishing, and epitaxial side and substrate metallization steps, the epilayer side was electroplated with an approximately $5-\mu \mathrm{m}$-thick gold layer. The wafer was then cleaved into 3- and 5-mm-long lasers, whose facets were subsequently high-reflection (HR) coated. Finally, the chips were mounted epilayer side down on aluminum nitride (AIN) or diamond submounts for pulsed and cw characterization. Pulsed testing was performed with 500 -ns pulses and $0.5 \%$ duty cycle.

Figure 3 shows the device performance in pulsed and cw modes at room temperature. These results were obtained using diamond as a submount because of its very high thermal conductivity. However, these are unsuitable for high-reliability operation of high-power lasers, because they require the use of a soft indium solder, due to the large coefficient of thermal expansion (CTE) mismatch between diamond and the InP QCL substrate. Recently, comparable power performance was obtained from 4.6- $\mu \mathrm{m}$ QCLs using an aluminum nitride CTE-matched submount and a hard AuSn solder, by optimizing the front facet's reflectivity and cavity length. ${ }^{9}$ Figure 4 illustrates the typical QCL package used by the Pranalytica-Harvard team.

The group of Prof. Razeghi at Northwestern University has also concentrated on high-power cw QCLs. ${ }^{12,13}$ They

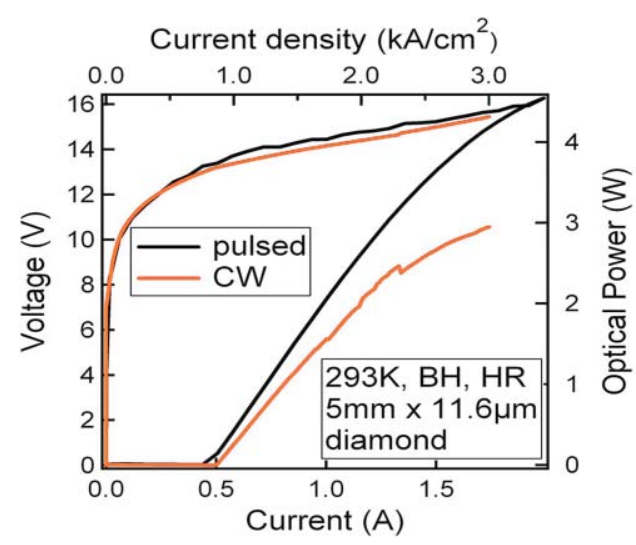

Fig. 3 Optical output power and $I-V$ characteristic in pulsed and $\mathrm{CW}$ mode of $\mathrm{a} \lambda \approx 4.6-\mu \mathrm{m} \mathrm{QCL}$. The box in the lower right corner indicates the operating temperature, device dimensions (cavity length times ridge width) and that a high reflection (HR) coating for the back facet was used. From Ref. 8. 


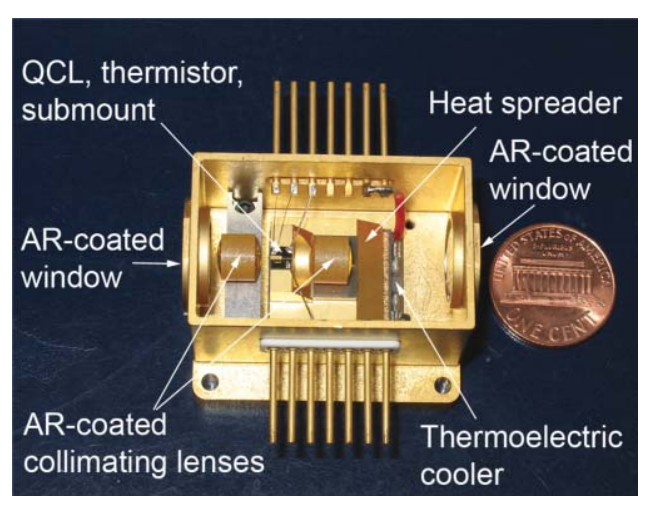

Fig. 4 Package for cw room-temperature QCL. From Ref. 8.

achieved comparable cw room-temperature performance to the Harvard-Pranalytica group. They grew an InP-based QCL heterostructure emitting at $\lambda \approx 4.6 \mu \mathrm{m}$, using gas-source molecular beam epitaxy. The wafer was fabricated into a double-channel ridge waveguide geometry with ridge widths of 19.7 and $10.6 \mu \mathrm{m}$ without semiinsulating InP regrowth. An uncoated, narrow ridge device with a $4.8-\mathrm{mm}$ cavity length was bonded epilayer down to a diamond submount and exhibited 2.5-W maximum output power with a wall plug efficiency of $12.5 \%$ at room temperature in $\mathrm{cw}$ operation.

AlInAs/GaInAs grown on InP substrate is the material of choice for mid-IR QCLs. Originally QCLs were produced only using MBE, a technique in wide use for the manufacture of many semiconductor devices. For growth of high-performance QCL heterostructures, several requirements must be satisfied. Foremost among these is the ability to form atomically abrupt interfaces between layers of nanometer or even subnanometer thickness. MBE is exemplary in this regard; however, a properly designed metalorganic chemical vapor deposition (MOCVD) reactor can approach the interface abruptness associated with MBE. Note that the interface abruptness will broaden the gain spectrum, leading to a lower peak gain and increased intersubband absorption due to nonresonant transitions. Both effects will increase the laser threshold. ${ }^{8}$

Low impurity-background doping in the active region, in concert with controlled intentional-doping profiles in the injector regions, is also critical for QCLs in order to minimize the broadening of the laser transition and thus reduce the laser threshold. This requirement is not a problem for the AlInAs and GaInAs alloys grown by MOCVD. In addition, MOCVD may even offer some advantages over MBE for QCL growth. Among them are stability of growth rate and composition over very long growth runs, the ease of achieving low oxygen contamination in aluminum-containing materials, the ability to grow thick InP cladding and burying layers, and the potential for very high growth rates. On the other hand, it is expected that interface formation will be more challenging for MOCVD; also, the requirement for organic precursors might lead to unacceptably high carbon background for some materials, such as AlGaAs/GaAs. Detailed studies of MOCVD grown QCLs operating at 4.6-, 5.2-, and 8.3- $\mu \mathrm{m}$ wavelength have shown that their pulsed and $\mathrm{cw}$ performance at room temperature is comparable to that of MBE QCLs. ${ }^{14,15} \mathrm{Re}-$ liability tests indicate that MOCVD QCL devices operated without degradation for more than $5000 \mathrm{~h}^{14}$

\section{Single-Mode Quantum Cascade Lasers}

In a distributed feedback (DFB) laser, the diffraction grating, which selects a single mode, is etched either on the upper surface of the laser ridge or in the material just above the injector-active-region stack on which a cladding layer is subsequently regrown. ${ }^{16}$ The period of the grating, $d$, determines the precise wavelength or laser mode that satisfies the Bragg condition $\lambda=2 n_{\text {eff }} d$, where $n_{\text {eff }}$ is the effective refractive index of the waveguide. Light satisfying this condition is strongly reflected off the grating and is selected for laser action. Wavelength tuning in these DFB QCLs is achieved by increasing the temperature of the laser either with a temperature controller or by using a sawtooth current waveform to drive the laser. Raising the temperature increases $n_{\text {eff }}$ and therefore the emission wavelength that satisfies the Bragg condition. Thus, the latter can be positioned in the vicinity of an absorption feature of interest, blue-shifted with respect to its peak. A subsequent slow current ramp applied to the QCL can be used to scan the wavelength across the absorption line as result of Joule heating of the laser active region. By an additional modulation of the current using a small sine wave signal of frequency much larger than the ramp, one can measure the derivative of the spectrum, leading to enhanced sensitivity. Figure 5 shows the temperature tuning of the emission wavelength of different DFB QCLs designed to match absorption lines of compounds, which overlap with one of the atmospheric windows. This overlap allows one to achieve high sensitivity (from a few parts per billion to as low as parts per trillion by volume) in the detection of trace gases in the atmosphere using laser absorption spectroscopy.

\section{Broadband Quantum Cascade Lasers}

The route to more applications required the production of QCLs with broader gain profiles and large single-mode tuning range that operated near room temperature. In the last few years, such QCLs have become available and have been incorporated into external cavities (ECs) to produce tuning ranges with a single QCL ${ }^{17-22}$ of $\approx 200 \mathrm{~cm}^{-1} \mathrm{cw}$ and more than $300 \mathrm{~cm}^{-1}$ pulsed. In a very recent development, an array of 32 DFB lasers on a single chip covering about $100 \mathrm{~cm}^{-1}$ in pulsed mode has been reported, ${ }^{23}$ making possible the

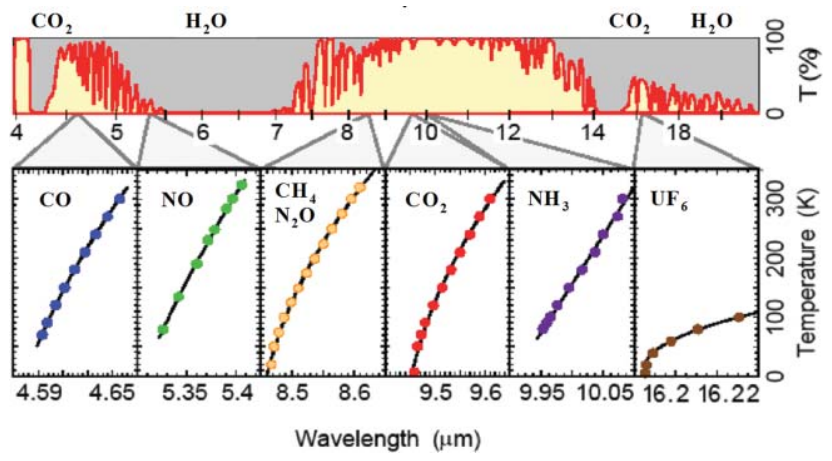

Fig. 5 The upper panel is the transmission spectrum at sea level of the atmosphere under standard conditions (room temperature and one atmosphere of pressure) over a path of about one kilometer. Note the two transmission windows below $5 \mu \mathrm{m}$ and between 8 and $13 \mu \mathrm{m}$. The lower panel represents the temperature tuning range of distributed feedback quantum cascade lasers designed to operate in selected regions of the transparency windows where many common gases have absorption fingerprints. From Ref. 4. 


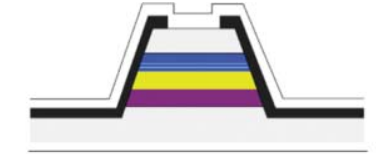

Broadband laser: each cascade designed for a different wavelength

(a)

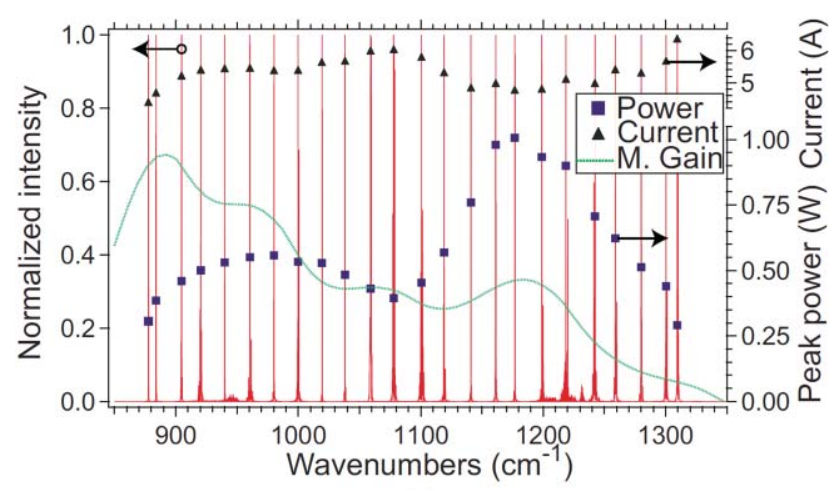

(c)

Fig. 6 Broadband quantum cascade lasers. (a) Schematic of multicascade configuration; each cascade in general comprises several laser stages (injector plus active region). (b) Schematic of external cavity tuning with grating which provides tuning by change of the angle $\theta$. (c) Demonstration of operation over $432 \mathrm{~cm}^{-1}$. (From Ref. 28.)

creation of broadly tunable subminiature IR laser sources for spectroscopy. The tuning range of this source was recently increased to $220 \mathrm{~cm}^{-1}$ using a 24-laser array. ${ }^{24}$

Tunability over a much broader range than what a typical DFB QCL can offer is a highly desirable feature for trace gas analysis, for it enables the parallel detection of multiple chemicals and allows mapping of very broad absorption lines, such as those of liquids. In this section we analyze the most effective device design strategies to achieve such a goal.

\subsection{Broadband Single-Mode Tuning: External-Cavity Grating Spectrometer}

The key step in achieving broad tunability is the design of a laser with a broad gain spectrum. QCLs, unlike diode lasers, can easily be designed to emit at multiple and widely differing wavelengths by stacking active regions corresponding to different optical transitions that span a wide range of energies (Fig. 6). Early demonstrations concentrated on dual-wavelength lasing, ${ }^{25}$ followed by a QCL that emitted simultaneously a quasicontinuum of wavelengths between 5 and $8 \mu \mathrm{m} .{ }^{26}$ To ensure quasicontinuous emission, the stages were designed so as to achieve an optimally flat gain spectrum over the emission range of interest. More recently, broadband QCL development has concentrated on continuous single-mode tunability over the broadest possible range. In order to achieve that, two design principles have been utilized.

Consider first the aforementioned structure with multiwavelength stages and a gain per stage engineered to be as broad as possible. This broad gain can be achieved using a so-called bound-to-continuum QCL, in which the lower state of the laser transition is a relatively broad continuum consisting of closely spaced sublevels spanning an energy range greater than an optical phonon. The many laser transitions fan out with comparable oscillator strengths, giving rise to a broad spectrum. The application of an EC configuration allows selection of the QCL wavelength anywhere within the available QCL spectral gain without changing the chip temperature, thus significantly increasing the laser spectral coverage and allowing much more efficient utilization of the available QCL gain width. This is especially important for the QCL gain media designed to have intrinsically broader gain profiles. Frequency tunability from 961 to $1220 \mathrm{~cm}^{-1}$ (24\% of the center wavelength) of a pulsed QCL was achieved using a heterogeneous gain medium with a two-wavelength (8.4 and $9.6 \mu \mathrm{m}$ ) active region in a Littrow-type EC-QCL configuration. $^{27}$ With this laser, single mode tuning from 1045 to $1246 \mathrm{~cm}^{-1}$ was also achieved.

Recently, ${ }^{28}$ lasers using symmetric active-region designs with five different cascades centered at different wavelengths have demonstrated tunability from 7.6 to $11.4 \mu \mathrm{m}$ with a peak optical output power of $1 \mathrm{~W}$ and an average output power of $15 \mathrm{~mW}$ at room temperature. With a tuning range greater than $432 \mathrm{~cm}^{-1}$, this single-mode source covers a continuous emission range of more than $39 \%$ around the center frequency. However, the design of a high-gain broadband active region covering a significantly larger spectral range without any gaps in the spectral emission is a serious challenge, because using more cascades promises even wider tuning ranges, but makes the design more complex.

Typically in an EC laser, coarse wavelength tuning is achieved by rotating the grating, with the feedback needed for lasing arising from the first-order grating reflection. However, merely rotating the grating will not result in continuous tuning of the laser over a significant frequency range. Instead, the laser frequency will hop from cavity mode to cavity mode. In order to achieve continuous tuning, the cavity length and grating angle must track each other. The situation can be further complicated, in a laser with no antireflective (AR) coating, by the optical cavity formed by the laser itself. The effects of a laser cavity can be avoided by AR-coating the facet of the QCL facing the grating to eliminate optical feedback. Several schemes have been devised to track the cavity length with the grating angle. ${ }^{17,19}$

One reported scheme for wide tuning without mode hops was based on a quantitative coupled-mode analysis of the EC QC laser. ${ }^{17}$ Realizing this tuning scheme involves active and simultaneous adjustment of all three relevant degrees of freedom: grating angle (via precise grating rotation), EC length (via a piezoelectric-transducer-controlled optical element), and the optical length of the chip (via driving current and/or chip temperature). This tuning scheme utilized a lookup table to identify the correct parametric values of all three variables as a function of the desired wavelength. Another scheme ${ }^{19}$ used a somewhat different approach of actively and simultaneously adjusting two degrees of freedom: the QCL chip optical length and the grating angle. A closed-loop servo with feedback was used to optimize the cavity length to select and support a desired single mode at every grating angle. In a more recent scheme ${ }^{29}$ the grating motion is mechanically constrained to follow a trajectory allowing for the simultaneous tuning of the diffraction grating angle and the laser cavity length, thereby constraining the EC mode to coincide with the wavelength selected by the grating. To avoid problems caused by the laser-cavity interaction, a QCL gain chip facet AR coating was developed with sufficiently low reflectivity $(<0.001)$ to suppress the residual étalon effect. Daylight 


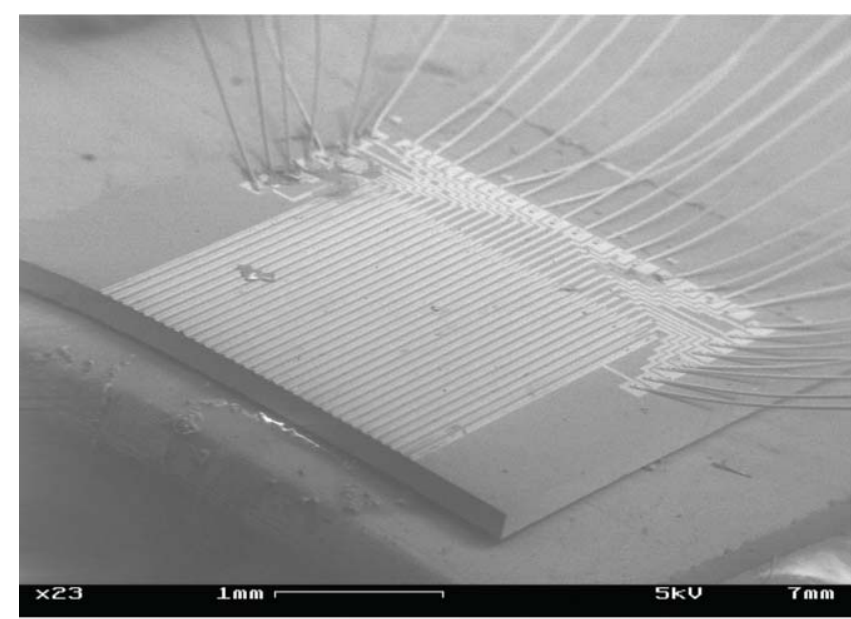

(a)

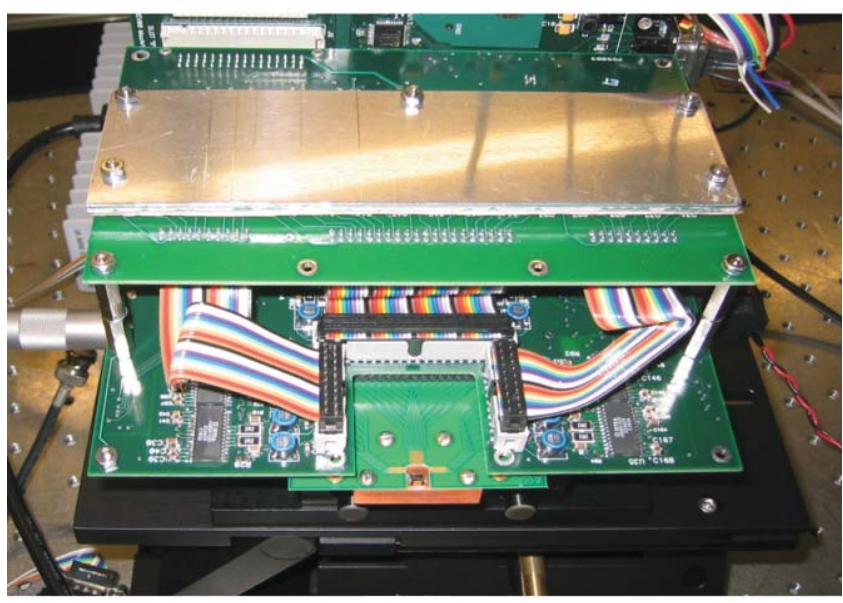

(b)

Fig. 7 (a) Broadband spectrometer on a chip, consisting of an array of QCL distributed-feedback lasers (from Ref. 23). (b) The chip is housed in a custom-designed microcontroller to drive the array. The width of the instrument is $20 \mathrm{~cm}$.

Solutions Inc. developed this design, and instruments based on it are commercially available.

\subsection{Broadband Multi-DFB Laser Spectrometer on a Chip}

Arrays of DFB QCLs can be made as single-mode sources covering a wide range of mid-IR frequencies. For example, a DFB-QCL array has been demonstrated ${ }^{23}$ that achieved single-mode lasing coverage of $85 \mathrm{~cm}^{-1}$ near $9 \mu \mathrm{m}$, using a bound-to-continuum active-region design. The devices were fabricated monolithically on the same chip and driven individually by a microelectronic controller [Fig. 7(a)]. The spacing of the emission wavelengths of the lasers in the array was sufficiently small that for any wavelength within the gain spectrum of the QC laser material, one could select a device in the array and adjust its temperature to produce single-mode emission at the desired frequency.

A custom-designed controller was employed, which consisted of pulse generators to power the lasers, direct current bias circuitry to heat individual lasers in the array for temperature tuning, and a serial port interface for computer control of laser firing [Fig. 7(b)]. Figure 8 shows the spectra of

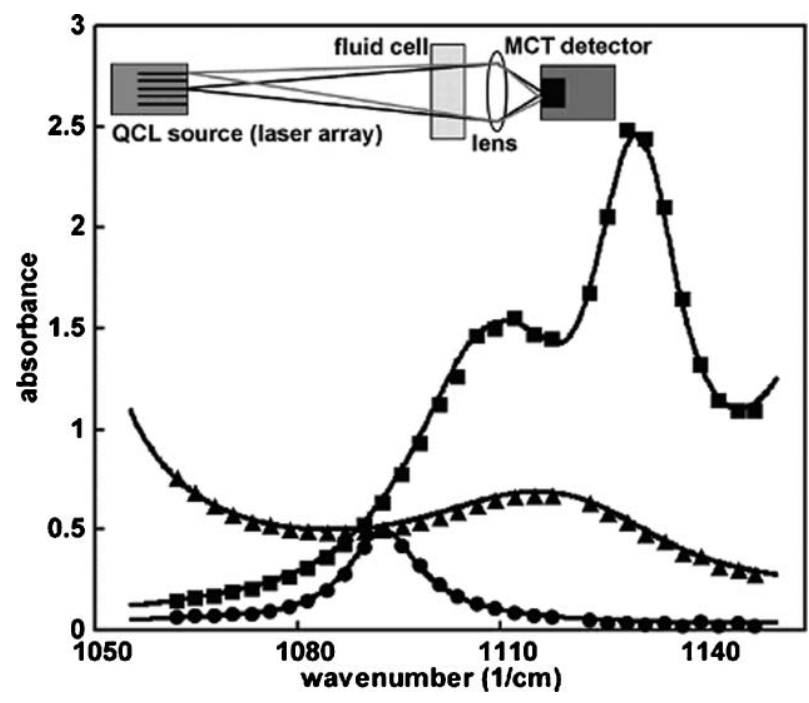

Fig. 8 Absorption spectra of isopropanol (squares), methanol (triangles), and acetone (circles) obtained with an on-chip spectrometer consisting of an array of 32 distributed-feedback quantum cascade lasers and with a Bruker Vertex 80v Fourier transform infrared spectrometer (continuous lines). The lasers were operated in a pulsed mode at room temperature. From Ref. 23. Inset: Experimental setup for midinfrared spectroscopy of liquids with the quantum cascade laser source.

several liquids obtained with this array. It took approximately 10 s to obtain each spectrum. ${ }^{23}$ The results compare favorably with spectra obtained using a conventional Fourier transform infrared (FTIR) spectrometer, also shown in Fig. 8

In order to obtain continuous spectral coverage between the nominal emission frequencies of the individual lasers in the array, one can tune the lasers in a small range with temperature. The lasers can be heated locally by applying a subthreshold dc current to tune an individual laser, or the lasers can be heated globally by changing the temperature of the heat sink on which the laser array chip sits. With dc bias current, the tuning was $5 \mathrm{~cm}^{-1}$ with $300 \mathrm{~mA}$. Alternatively, the same tuning was achieved by varying the heat-sink temperature with a thermoelectric cooler from 252 to $325 \mathrm{~K}$. Local heating using dc current can achieve the desired temperature in milliseconds, while heat-sink temperature changes typically take tens of seconds. We note that the frequency resolution of this QCL spectrometer is determined by the lasers' linewidth, which was measured to be $\sim 0.01 \mathrm{~cm}^{-1}$ in pulsed operation and $\sim 0.001 \mathrm{~cm}^{-1}$ in $\mathrm{cw}$ operation. This is significantly better than the resolution offered by a typical bench-top FTIR spectrometer $\left(\sim 0.1 \mathrm{~cm}^{-1}\right)$. Despite the narrower spectral measurement range than those of FTIR spectrometers, we believe that this QCL-based spectrometer can provide a portable alternative to FTIR spectrometers in significant portions of the midIR molecular fingerprint region. The fact that it uses lasers instead of a Globar (the thermal source used in FTIR spectrometers), has great advantages in terms of much higher brightness, which could be of paramount important in standoff detection (e.g., in the measurement of a spectrum reflected off a surface or aerosol clouds) for remote sensing of trace gases. Recently, wavelength beam combining was used to copropagate the multiple beams from the array of DFB QCLs. ${ }^{30}$ The beam-quality product of the array, defined as the product of near-field spot size and far-field divergence for the 
entire array, was improved by a factor of 21 by using wavelength beam combining. To demonstrate the applicability of wavelength-beam-combined DFB QCLs arrays for remote sensing, the absorption spectrum of isopropanol was measured at a distance of $6 \mathrm{~m}$ from the array. ${ }^{30}$ Beam combining was accomplished by a suitably placed grating and transform lens that overlap the beams from each laser in both near field and far field.

In recent work, ${ }^{24}$ the range of frequencies covered by a single DFB QC laser spectrometer was greatly increased to cover the available gain spectrum. The emission spectrum of the array (Fig. 9) covers a range of $210 \mathrm{~cm}^{-1}$, much broader than that of the original spectrometer $\left(85 \mathrm{~cm}^{-1}\right){ }^{23}$ Shown also are the spectrum of an individual device, indicating its single-mode character, and a micrograph of the device cross section.

\section{Short-Wavelength Mid-IR QCLs}

The upper frequency limit of QCLs is determined by the maximum barrier heights defining the quantum wells. Reaching wavelengths below $4 \mu \mathrm{m}$ has proven to be very challenging. ${ }^{31-36}$ However this situation may soon change, for research in this field is active in several groups. ${ }^{33-35,37-42}$ In the most widely used AlInAs/GaInAs material system, a short-wavelength midinfrared QCL, emitting pulsed at $3.5 \mu \mathrm{m}$ (at $280 \mathrm{~K}$ ), was demonstrated using straincompensated AlInAs/GaInAs on InP substrates. ${ }^{31}$ Shorter wavelengths down to $3.0 \mu \mathrm{m}$ are possible, but at a cost of substantially lower temperatures. Several very recent breakthroughs in pulsed room-temperature operation toward $3 \mu \mathrm{m}$ have been described, based on using heterostructures with very high conduction-band offsets: InAs-AlSb on InAs substrates $^{37}$ and InGaAs-AlAs(Sb) on InP substrates. ${ }^{31,40}$

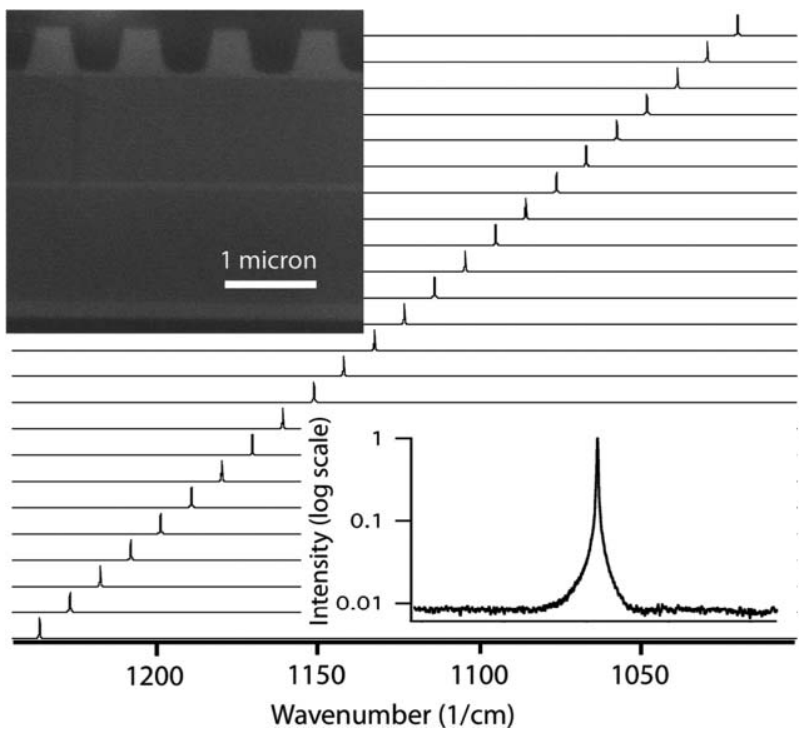

Fig. 9 Spectra of an array of 24 single-mode DFB quantum cascade lasers. Laser frequencies are spaced $\approx 9.5 \mathrm{~cm}$ apart and span a range of $\approx 220 \mathrm{~cm}$. Inset, top left: Scanning electron micrograph showing a cross section of the device, which has been cut along the laser ridge. The grating corrugation can be seen as the rectangular wave near the top of the image, and the two active regions are below, with a thin InGaAs spacer between them. Inset, bottom right: Spectrum of a representative laser in the array on a log scale, showing side-mode suppression greater than 100. From Ref. 24.
In Ref. 33, QCLs emitting at a wavelength near 3.3 $\mu \mathrm{m}$ up to a temperature of $400 \mathrm{~K}$ in pulsed mode were reported. Recently, a pulsed room-temperature singlemode-operation DFB QCL at a wavelength as short as 3.34 $\mu \mathrm{m}\left(\approx 3000 \mathrm{~cm}^{-1}\right)$ with an output power of $0.8 \mathrm{~W}$ has been demonstrated. ${ }^{39}$ QCLs emitting at wavelengths near $\lambda=3.1 \mu \mathrm{m}\left(\approx 3225 \mathrm{~cm}^{-1}\right)$ at room temperature have been demonstrated. ${ }^{42}$ The lasers operated in pulsed mode with an optical power of $8 \mathrm{~mW}$ at $295 \mathrm{~K}$. By cooling to cryogenic temperatures, wavelengths as short as $2.75 \mu \mathrm{m}$ $\left(\approx 3600 \mathrm{~cm}^{-1}\right)$ have been demonstrated. ${ }^{33}$

In the 3- to 4- $\mu \mathrm{m}$ spectral region there is another technology that has demonstrated significant performance improvements, the interband cascade laser. The concept was pioneered by R. Q. Yang ${ }^{43}$ in 1995 and is based on an interband optical transition between the valence band of $\mathrm{GaSb}$ and the conduction band of an adjacent InAs layer, separated from the GaSb by a thin AlSb tunnel barrier. What makes this optical transition possible is the broken-gap band alignment of the GaSb/InAs heterojunction, whereby the top of the valence band of the former is above the conduction-band bottom of the latter. This band arrangement permits cascading of many identical stages, just as in QCLs, so that an electron can sequentially tunnel through multiple stages, emitting a laser photon at each stage, and thus increasing the optical power. The best result reported so far with this technology is $\mathrm{cw}$ operation at $300 \mathrm{~K}$ for a $\lambda=3.75-\mu \mathrm{m}$ device with an optical power in excess of $10 \mathrm{~mW}^{44}$

\section{Noise and Linewidth of Quantum Cascade Lasers}

To achieve ultimate resolution in spectroscopy the linewidth of the laser source must be known. Recently the frequencynoise power spectral density of QCLs has been measured for the first time, using an ultralow-technical-noise laser current source. ${ }^{45}$ This allowed for the first time a study of the intrinsic linewidth of a QCL. They measured the frequencynoise power spectral density of a free-running mid-IR $(\lambda \approx 5 \mu \mathrm{m})$ single-mode cryogenically cooled QCL, using the side of a Doppler-broadened molecular transition of $\mathrm{CO}_{2}$. This well-established method allows one to convert laser frequency fluctuations into detectable intensity variations with negligible added noise. ${ }^{46}$ The spectrum of the intensity transmitted by this discriminator, when the laser frequency is locked to the half-height position (i.e., where the small-signal response is linear), reproduces the spectrum of the laser frequency fluctuations "amplified" by the slope of the absorption profile. The signal was detected with a fast 200-MHz-bandwidth $\mathrm{HgCdTe}$ detector. For these measurements, a current driver with ultralow noise (approaching shot noise) was developed, thus enabling the measurement of the intrinsic laser noise. Between 10 and $100 \mathrm{MHz}$, a white noise plateau is observed, which decreases with increasing drivecurrent-to-threshold-current ratio $\left(I / I_{\text {th }}\right)$ and corresponds to an intrinsic QCL linewidth of $510 \mathrm{~Hz}$ for $I / I_{\mathrm{th}}=1.54$ and a $\mathrm{cW}$ optical power of $6 \mathrm{~mW}$. At lower frequencies the noise showed two regimes of $1 / f$ and $1 / f^{2}$ dependence.

The observed hyperbolic dependence on $I / I_{\text {th }}$ is in qualitative agreement with recent theoretical predictions of the QCL linewidth. ${ }^{47}$ For $I / I_{\text {th }}=1.54$ its value is about $20 \%$ larger than the measured valued, a discrepancy that could be in part explained from uncertainties in the active-region parameters used in the calculation, such as lifetimes and 
the upper-laser-level injection efficiency. ${ }^{47}$ The latter had been previously assumed to be governed by the standard Schawlow-Townes formula of laser theory, augmented by the factor $1+\alpha^{2}$, where $\alpha$ is the alpha parameter, which represents the effect of refractive index variations caused by electron density fluctuations. In QCLs, however, these variations are negligible at the peak of the gain spectrum, because the latter is in most cases symmetric (Lorentzian). This fact led the authors of Ref. 3 to predict that $\alpha$ in QCLs should be very small. Several subsequent measurements confirmed that. ${ }^{48-51}$ The remarkable feature of Ref. 47 is that it predicts, for small $\alpha$, linewidths even narrower than the SchawlowTownes value $\left(\Delta_{\mathrm{ST}}\right)$, by a factor depending on $I / I_{\text {th }}$ and on the ratio of the spontaneous emission rate coupled into the laser mode to the total relaxation rate of the upper level. In most lasers, the latter is dominated by spontaneous emission, which is the fundamental source of noise. The fact that, in QCLs, the nonradiative relaxation rate from the upper level is instead many orders of magnitude larger than the spontaneous rate from the same level leads to the reduction of the laser linewidth well below the Schawlow-Townes limit. ${ }^{47}$ Note that in the experiments described, the measured QCL linewidth is substantially below the linewidth $(4 \mathrm{kHz}$ at a laser power of $6 \mathrm{~mW}$ ) calculated using the Schawlow-Townes formula.

\section{Commercialization of QCLs}

In the short span of fifteen years QCLs have gone from a lab curiosity to the role of major players in the high-power and chemical sensing sector. To date one counts over 15 companies commercializing QCLs at the wafer, device, and system levels. Among the most significant players commercializing QCLs are Hamamatsu and an Alcatel Lucent-Thales venture (III-V Laboratories). Daylight Solutions sells tunable external-cavity QCLs, and Pranalytica Inc. high-power QCLs for countermeasures as well as beacons and illuminators. Aerodyne Inc. is specializing in QCL-based sensors for atmospheric chemistry. For a complete list of companies and an in-depth review of spectroscopic application of QCLs to chemistry the reader is referred to a recent article. $^{52}$

\section{Acknowledgments}

The author acknowledges collaborations with B. Lee, M. A. Belkin, L. Diehl, C. Pfluegl, Q. J. Wang, A. Lyakh, R. Maulini, A. Tsekoun, C. K. N. Patel, A. Y. Cho, D. L. Sivco, J. Faist, C. Sirtori, C. Gmachl, A. L. Hutchinson, R. F. Curl, and F. Tittel and support from DARPA and the Air Force Office for Scientific Research.

\section{References}

1. S. Mukherjee, and Z. S. Shi, "State of art of IV-VI semiconductor light emitting devices in mid infrared opto-electronic applications," IETE Tech. Rev. 26, 236-245 (2009).

2. R. H. Rediker, I. Melngailis, and A. Mooradian, IEEE J. Quantum Electron. 20, 602 (1984).

3. J. Faist, F. Capasso, D. L. Sivco, A. L. Hutchinson, and A. Y. Cho, Science 264, 553 (1994).

4. C. Gmachl, F. Capasso, D. L. Sivco, and A. Y. Cho, Rep. Prog. Phys. 64, 1533 (2001)

5. F. Capasso, C. Gmachl, R. Paiella, A. Tredicucci, A. L. Hutchinson, D. L. Sivco, J. N. Baillargeon, A. Y. Cho, and H. C. Liu, IEEE J. Sel. Top. Quantum Electron. 6, 931 (2000).

6. M. Razeghi, IEEE J. Sel. Top. Quantum Electron. 15, 941 (2009).
7. M. Razeghi, Proc. SPIE 7230, 723011 (2009).

8. A. Lyakh, R. Maulini, A. Tsekoun, R. Go, C. Pflugl, L. Diehl, Q. J. Wang, F. Capasso, and C. K. N. Patel, Appl. Phys. Lett. 95, 141113 (2009).

9. R. Maulini, A. Lyakh, A. Tsekoun, R. Go, C. Pflugl, L. Diehl, F. Capasso, and C. K. N. Patel, Appl. Phys. Lett. 95, 151112 (2009).

10. D. Hofstetter, M. Beck, T. Aellen, and J. Faist, Appl. Phys. Lett. 78, 396 (2001).

11. M. Beck, D. Hofstetter, T. Aellen, J. Faist, U. Oesterle, M. Ilegems, E. Gini, and H. Melchior, Science 295, 301 (2002).

12. Y. Bai, S. R. Darvish, S. Slivken, W. Zhang, A. Evans, J. Nguyen, and M. Razeghi, Appl. Phys. Lett. 92, 101105 (2008).

13. Y. Bai, S. Slivken, S. R. Darvish, and M. Razeghi, Appl. Phys. Lett. 93 021103 (2008).

14. M. Troccoli, L. Diehl, D. P. Bour, S. W. Corzine, N. F. Yu, C. Y. Wang, M. A. Belkin, G. Hofler, R. Lewicki, G. Wysocki, F. K. Tittel, and F. Capasso, J. Lightw. Technol. 26, 3534 (2008).

15. A. Lyakh, C. Pflugl, L. Diehl, Q. J. Wang, F. Capasso, X. J. Wang, J. Y. Fan, T. Tanbun-Ek, R. Maulini, A. Tsekoun, R. Go, and C. K. N. Patel, Appl. Phys. Lett. 92, 111110 (2008).

16. J. Faist, C. Gmachl, F. Capasso, C. Sitori, D. L. Sivco, J. N. Baillargeon, A. L. Hutchinson, and A. Y. Cho, Appl. Phys. Lett. 70, 2670 (1997).

17. G. Wysocki, R. F. Curl, F. K. Tittel, R. Maulini, J. M. Bulliard, and J. Faist, Appl. Phys. B 81, 769 (2005).

18. R. Maulini, A. Mohan, M. Giovannini, J. Faist, and E. Gini, Appl. Phys. Lett. 88, 201113 (2006)

19. M. B. Pushkarsky, I. G. Dunayevskiy, M. Prasanna, A. G. Tsekoun, R. Go, and C. K. N. Patel, Proc. Natl. Acad. Sci. U.S.A. 103, 19630 (2006).

20. M. Pushkarsky, A. Tsekoun, I. G. Dunayevskiy, R. Go, and C. K. N. Patel, Proc. Natl. Acad. Sci. U.S.A. 103, 10846 (2006).

21. G. Wysocki, R. Lewicki, R. F. Curl, F. K. Tittel, L. Diehl, F. Capasso, M. Troccoli, G. Hofler, D. Bour, S. Corzine, R. Maulini, M. Giovannini, and J. Faist, Appl. Phys. B 92, 305 (2008).

22. A. Wittmann, A. Hugi, E. Gini, N. Hoyler, and J. Faist, IEEE J. Quantum Electron. 44, 1083 (2008).

23. B. G. Lee, M. A. Belkin, C. Pfugl, L. Diehl, H. F. A. Zhang, R. M. Audet, J. MacArthur, D. P. Bour, S. W. Corzine, G. E. Hofler, and F Capasso, IEEE J. Quantum Electron. 45, 554 (2009).

24. B. G. Lee, H. F. A. Zhang, C. Pflugl, L. Diehl, M. A. Belkin, M. Fischer, A. Wittmann, J. Faist, and F. Capasso, IEEE Photon. Technol. Lett. 21, 914 (2009).

25. C. Gmachl, D. L. Sivco, J. N. Baillargeon, A. L. Hutchinson, F. Capasso, and A. Y. Cho, Appl. Phys. Lett. 79, 572 (2001).

26. C. Gmachl, D. L. Sivco, R. Colombelli, F. Capasso, and A. Y. Cho, Nature 415, 883 (2002)

27. A. Wittmann, A. Hugi, S. Blaser, M. Giovanni, and J. Faist, Optics Lett. 32, 2792 (2007).

28. A. Hugi, R. Terazzi, Y. Bonetti, A. Wittmann, M. Fischer, M. Beck, J. Faist, and E. Gini, Appl. Phys. Lett. 95, 061103 (2009).

29. M. Pushkarsky, M. Weida, T. Day, D. Arnone, R. Pritchett, D. Caffey, and S. Crivello, Proc. SPIE 6871, 68711X (2008).

30. B. G. Lee, J. Kansky, C. Goyal, C. Pfluegl, L. Diehl, M. A. Belkin, A. Sanchez, and F. Capasso, Opt. Express 17, 16216 (2009).

31. J. Faist, F. Capasso, D. L. Sivco, A. L. Hutchinson, S. N. G. Chu, and A. Y. Cho, Appl. Phys. Lett. 72, 680 (1998).

32. M. P. Semtsiv, M. Ziegler, S. Dressler, W. T. Masselink, N. Georgiev, T. Dekorsy, and M. Helm, Appl. Phys. Lett. 85, 1478 (2004).

33. J. Devenson, O. Cathabard, R. Teissier, and A. N. Baranov, Appl. Phys. Lett. 91, 251102 (2007).

34. X. Marcadet, C. Renard, M. Carras, M. Garcia, and J. Massies, Appl. Phys. Lett. 91, 161104 (2007).

35. M. P. Semtsiv, S. Dressler, and W. T. Masselink, IEEE J. Quantum Electron. 43, 42 (2007).

36. M. P. Semtsiv, M. Wienold, S. Dressler, and W. T. Masselink, Appl. Phys. Lett. 90, 051111 (2007).

37. J. Devenson, O. Cathabard, R. Teissier, and A. N. Baranov, Appl. Phys. Lett. 91, 141106 (2007).

38. J. P. Commin, D. G. Revin, S. Y. Zhang, A. B. Krysa, and J. W. Cockburn, Appl. Phys. Lett. 95, 111113 (2009).

39. O. Cathabard, R. Teissier, J. Devenson, and A. N. Baranov, Electron. Lett. 45, 1028 (2009).

40. J. S. Yu, S. Slivken, S. R. Darvish, and M. Razeghi, IEEE Photon. Technol. Lett. 17, 1154 (2005)

41. J. S. Yu, A. Evans, S. Slivken, S. R. Darvish, and M. Razeghi, Appl. Phys. Lett. 88, 251118 (2006).

42. S. Y. Zhang, D. G. Revin, J. W. Cockburn, K. Kennedy, A. B. Krysa, and M. Hopkinson, Appl. Phys. Lett. 94, 031106 (2009).

43. R. Q. Yang, Superlattices Microstruct. 17, 77 (1995).

44. M. Kim, C. L. Canedy, W. W. Bewley, C. S. Kim, J. R. Lindle, J. Abell, I. Vurgaftman, and J. R. Meyer, Appl. Phys. Lett. 92, 191110 (2008).

45. S. Bartalini, S. Borri, P. Cancio, A. Castrillo, I. Galli, G. Giusfredi, D. Mazzom, L. Gianfrani, and P. De Natale, Phys. Rev. Lett. 104, 083904 (2010). 
46. D. S. Elliott, R. Roy, and S. J. Smith, Phys. Rev A 26, 18 (1982).

47. M. Yamanishi, T. Edamura, K. Fujita, N. Akikusa, and H. Kan, IEEE J. Quantum Electron. 44, 12 (2008).

48. T. Aellen, R. Maulini, R. Terazzi, N. Hoyler, M. Giovannini, J. Faist, S. Blaser, and L. Hvozdara, Appl. Phys. Lett. 89, 091121 (2006).

49. J. Faist, F. Capasso, C. Sirtori, D. L. Sivco, A. L. Hutchinson, and A. Y. Cho, Appl. Phys. Lett. 67, 3057 (1995).

50. N. Kumazaki, Y. Takagi, M. Ishihara, K. Kasahara, A. Sugiyama, N. Akikusa, and T. Edamura, Appl. Phys. Lett. 92, 121104 (2008).

51. J. von Staden, T. Gensty, W. Elsäßer, G. Giuliani, and C. Mann, Opt. Lett. 31, 2574 (2006).

52. R. F. Curl, F. Capasso, C. Gmachl, A. A. Kosterev, B. McManus, R. Lewicki, M. Pusharsky, G. Wysocki, and F. K. Tittel, Chem. Phys. Lett. 487, 1 (2010).

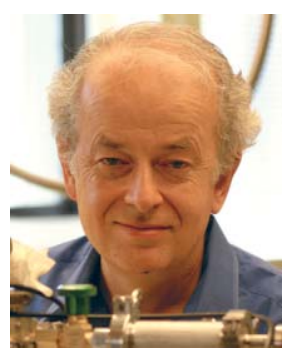

Federico Capasso is the Robert Wallace Professor of Applied Physics at Harvard University, which he joined in 2003 after a 27 years' career at Bell Labs, where he did research, became a Bell Labs Fellow, and held several management positions, including Vice President for Physical Research. His research has spanned a broad range of topics from applications to basic science in the areas of electronics, photonics, mesoscopic physics, nanotechnology, and quantum elec- trodynamics. $\mathrm{He}$ is a coinventor of the quantum cascade laser, a fundamentally new light source, which has now been commercialized, and in recent years he has been involved in fundamental studies of the Casimir force, including the first measurement of a repulsive Casimir force. He is a member of the National Academy of Sciences and the National Academy of Engineering, a fellow of the American Academy of Arts, and Sciences, and an honorary member of the Franklin Institute. His awards include the King Faisal International Prize for Science, the Berthold Leibinger Zukunftspreis (Future Prize), the American Physical Society Arthur Schawlow Prize, the IEEE Edison Medal, the Wetherill Medal of the Franklin Institute, the Optical Society of America Wood Prize, the Materials Research Society Medal, the Rank Prize in Optoelectronics, the IOP Duddell Medal, the Willis Lamb Medal, the IEEE David Sarnoff Award, the IEEE-LEOS Streifer Award, the LVMH Leonardo Da Vinci Prize, and the Welker Medal. He is a fellow of OSA, APS, IEEE, SPIE, IOP, and AAAS. 